\title{
Ecoeficiencia: clave de la responsabilidad ambiental empresarial en el sector textil
}

\author{
Eco-efficiency: The Key to Corporate Environmental \\ Responsibility in the Textile Industry
}

\author{
Remedios Pitre-Redondo \\ Universidad de la Guajira, Riohacha, Colombia. \\ Santander De la Ossa-Guerra (iD) \\ Universidad de Sucre, Sincelejo, Colombia. \\ Hugo Hernández-Palma iD \\ Universidad del Atlántico, Barranquilla, Colombia.
}

\section{Autor de Correspondencia}

hugoghernandezp@mail.uniatlant ico.edu.co

Recibido: 03-03-2020

Aceptado: 08-07-2020

Publicado: 30-11-2020

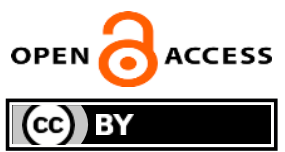

Copyright (C) 2020

Desarrollo Gerencial

\section{Resumen}

Objetivo: Este estudio tuvo como finalidad identificar si las pequeñas y medianas empresas textiles (PYMES), ubicadas en el Departamento del Atlántico, Colombia, realizan prácticas de Ecoeficiencia para el control de residuos contaminantes. Método: se utilizó una investigación de tipo descriptivo, con un enfoque cuantitativo y un diseño de campo no experimental, en la que se aplicó una encuesta cerrada, dirigida a una muestra de 53 empresas. Resultados y discusión: Entre los principales hallazgos se encontró que el $56 \%$ de las PYMES participantes no realizan acciones para reducir el volumen o la peligrosidad de sus residuos, ya que no los consideran importantes ni rentables. Por lo tanto, estos procesos no son ecoeficientes y el compromiso con la preservación del medio ambiente y la sostenibilidad de las PYMES en estudio es insuficiente. Conclusiones: Dado que el sector textil es uno de los recursos con menor índice de reutilización del mundo, es fundamental que este vínculo con los procesos de gestión sostenible y la ecoeficiencia sea claro y constante, apuntando a una internalización de los procesos de responsabilidad social, apoyándose en el Estado, las familias, las personas y las leyes para su constante evolución y desarrollo.

Palabras clave: Desarrollo sostenible, Prácticas ambientales, Responsabilidad social, Residuos sólidos.

Clasificación JEL: Q01, Q50, Q56.

\section{Abstract}

Objective: This study aimed to identify whether small and medium sized textile companies (SMEs), located in the Department of Atlántico, Colombia, currently carry out Eco-efficiency practices for the control of waste, contaminating residues, among other aspects. Method: A descriptive type of research was used, with a quantitative approach and a non-experimental field design, in which a closed survey was applied, directed at a sample of 53 companies. Results and discussion: Among the main findings, it was found that $56 \%$ of the participating SMEs do not take actions to reduce the volume or hazardousness of their waste, since they do not consider it important or profitable. Therefore, these processes are not eco-efficient and the commitment to environmental preservation and sustainability of the SMEs under study is insufficient. Conclusions: Given that the textile sector is one of the resources with the lowest rate of reuse in the world, it is fundamental that this link with the processes of sustainable management and eco-efficiency be clear and constant, aiming at an internalization of the processes of social responsibility, relying on the State, families, people and laws for their constant evolution and development.

Keywords: Sustainable development, Social responsibility, Environmental practices, Solid waste. JEL classification: 001 . 050. 056.

Como citar este artículo (APA):

Pitre-Redondo, R., De la Ossa-Guerra, S., \& Hernández-Palma, H. (2020). Ecoeficiencia: clave de la responsabilidad ambiental empresarial en el sector textil. Desarrollo Gerencial, 12(2), 1-20. https://doi.org/10.17081/dege.12.2.3870 


\section{Introducción}

Con el paso de los años, el ser humano ha pasado de ser una especie nómada el cual se dedicaba a la casa y a los cultivos estacionarios a convertirse hoy en día en seres sedentarios; caracterizados por el establecimiento de comunidades y el desarrollo de industrias a gran escala para lograr suplir las necesidades de sus iguales. A partir de este punto, es innegable reconocer el actual impacto que ha tenido esta tendencia y estilo de vida en el medio ambiente; pues el cambio climático con el pasar de cada segundo está teniendo mayores connotaciones y trayendo consecuencias que podrían llegar a ser irreversibles si no se toman las acciones necesarias a tiempo. Basta con revisar los números de emisiones de CO2 (Dióxido de carbono), que al sol de hoy han alcanzado los números más altos en los últimos 800.000 años, según reportes de Oceanografía Scripps (2019) y al año 2007 según reportes de Intergovernmental Panel on Climate Change (2007), el $19 \%$ de esta cifra corresponde a la responsabilidad del sector industrial.

En tal sentido, la sociedad actual está tomando conciencia acerca del impacto que tiene la industria sobre el deterioro del medio ambiente; según Suárez y Molina (2014), el desarrollo industrial si bien induce una fuerte reactivación socioeconómica, contribuye a la generación de diversas problemáticas ambientales como es el caso de la contaminación, el desequilibrio en los ecosistemas y la extinción de fauna y flora y, así mimo, da paso a la génesis de problemáticas sociales, entre ellas y quizás la más relevante, el detrimento de la salud pública principalmente, a partir de la contaminación atmosférica (Ballester, et. al., 2003; Vargas, 2005) y de la contaminación en las cuencas hídricas (Maldonado, 2009)

Este hecho viene como resultado de un extenso proceso histórico por el cual ha pasado la sociedad para poco a poco ir reconociendo el cambio climático como una problemática real, la cual es resultado de la acción indiscriminada del hombre y de sus industrias que por mucho tiempo se desarrollaron obviando las necesidades y el impacto hacia los ecosistemas.

Lo anterior, ha sido el punto de partida para la creación de leyes proteccionistas que impulsan estrategias direccionadas a la evaluación, prevención y control de los factores desligados de la actividad industrial, que influyen en la aparición de problemas ambientales (Torres, Barreto y Rincón, 2015). Pero así mismo, ha motivado a las empresas que desean ser competitivas a desarrollar sistemas de gestión que les ayuden a reducir su huella ambiental, por medio del mejoramiento de sus procesos, la adquisición de tecnológicas y prácticas de producción limpias, el posicionamiento de políticas de optimización de recursos e incluso, el eco-diseño de sus productos; todo ello con la finalidad de hacer un uso efectivo de sus recursos, reducir costes y eco-posicionarse en el mercado. 
El eco-posicionamiento, según la Fundación Fórum Ambiental (2014) se ha popularizado entre las empresas por cuestiones como la calidad, el ahorro, el mercado, la imagen, las oportunidades de negocio $y$, las consideraciones de tipo ético y social.

De acuerdo con Eryuruk (2012) las empresas de hoy deben rediseñar sus estructuras existentes, para convertirse en ambientalmente responsables, de este modo puedan aumentar su credibilidad en el medio pero, así mismo, producir más, con menos recursos, lo que evita el desperdicio de las materias primas, disminuye las emisiones y reduce los daños al medio ambiente (Mansour et. al., 2006).

Uno de los sectores industriales con mayor grado de contaminación ambiental en Colombia, es el textil; de acuerdo con el informe de Colombia Productiva (2012) la mayoría de las empresas de este sector no cuentan con políticas de sostenibilidad ni de Responsabilidad Social Empresarial RES ya que el $67 \%$ de estas empresas no elabora ningún tipo de informe de sostenibilidad, el $25 \%$ no tiene gestión de aguas residuales, el $58 \%$ no cuenta con un sistema de control de emisiones atmosféricas y solo el $4,4 \%$ de las empresas recuperan o reutilizan residuos de su producción. Estas cifras son alarmantes debido a que la producción textil en Colombia sigue en aumento, mientras que la mayoría de estas empresas adolecen de iniciativas para reducir su impacto medioambiental, siendo evidentes factores de riesgo desligados de si operación, como es el caso de los residuos químicos altamente contaminantes.

En atención a lo anterior, surge el concepto de Ecoeficiencia, una práctica que intenta sostener el valor ecológico de las empresas al tiempo que potencia el crecimiento económico del mercado; así pues, las empresas que implementan prácticas de Ecoeficiencia consiguen aumentar su competitividad, contribuyendo, al tiempo, al desarrollo sostenible del territorio, puesto que, añaden mayor valor a sus productos con un menor consumo de recursos naturales y disminuyen la contaminación, lo que ayuda a mitigar los riesgos ambientales que pueden llegar a afectar a sus stakeholders internos y externos.

En este sentido, se destaca que las prácticas relacionadas con la preservación del ambiente por parte de las organizaciones vienen como resultados de lo que se llama la Responsabilidad Social Empresarial RSE, lo cual es un propósito importante para toda organización, así como para los grupos de interés, pues permite lograr sus objetivos de mejor manera y tener un impacto positivo en el entorno y es una serie de obligaciones y compromisos tanto legales, como éticos, nacionales e internacionales que adquieren las empresas con los llamados grupos de interés, derivados del impacto generado por la existencia, actividad, además las operaciones de las organizaciones, las cuales son producidas en el contexto social, laboral, medioambiental, así como también al relacionado a los derechos humanos, en tal aspecto, este tiene un efecto significativo en el proceso de gestión de las empresas, tanto a lo relacionado a sus diversas actividades productivas y comerciales, así como en el desarrollo de las relaciones con los mencionados grupos de interés (Duran, Fuenmayor, Sergio y Hernández, 2016). 
Bajo estas ideas, se aporta que actualmente Colombia es una nación que dentro del marco de la sostenibilidad se encuentra atrasado con relación a otros países, pues no se toman en cuenta diversos procesos o regulaciones que permitirían asegurar la prevalencia de los ecosistemas en el país (Cuartas y Méndez, 2016).

A demás, desde ciertas posturas, se aporta que brindar especial atención a las necesidades del medio ambiente sobre las necesidades de las organizaciones puede traer como resultados un declive de la calidad competitiva de dichas empresas frente a los mercados; trayendo como resultado un declive en los índices económicos y laborales de los países y regiones. Ciertamente, se debe tener en cuenta que hoy en día se vive una época caracterizada por los mercados altamente competitivos; donde los constantes avances tecnológicos por resultado de la globalización obligan a las empresas a siempre buscar la innovación y la calidad como premisa para mantener su estatus competitivo (Parra et al, 2018).

A partir de este planteamiento, surge la disyuntiva de encontrar un equilibrio entre la calidad de los procesos productivos de manera que se tengan en cuenta las necesidades del medio ambiente a través de procesos de gestión sostenible; concepto direccionado a la gestión de los recursos encaminado a la calidad de los procesos cuidando la necesidad de los ecosistemas (Custodio y De Oliveira, 2016)

A partir de lo anterior se considera que la Ecoeficiencia es una alternativa para alcanzar este valor de posicionamiento dentro de los mercados, tomando en consideración el cambio climático como hecho catalizador de acciones enfocadas a la sostenibilidad. De esta manera, el presente artículo de investigación se desarrolló con la finalidad de identificar si las pequeñas y medianas empresas (Pymes) textiles, ubicadas en el departamento del Atlántico, Colombia en la actualidad llevan a cabo prácticas de Ecoeficiencia para el control de despilfarros, desechos contaminantes, consumo de energía, entre otros; aspectos que apuntan al posicionamiento de prácticas ambientalmente responsables. Si bien, según la Superintendencia de Sociedades (2013), el departamento del Atlántico solo aporta el 3,62\% de la producción textil nacional, se selecciona este segmento geográfico para el estudio, dada la evolución que este ha tenido en los últimos años en el sector textil, además, porque, a partir de su posición privilegiada y vocación exportadora, el departamento del Atlántico ha dado paso a dos importantes clústeres: uno de confecciones y otro diseño textil.

\section{Fundamentación Teórica}

\section{Responsabilidad Social Empresarial RSE}

La RSE es uno de los elementos más representativos de las organizaciones en la actualidad pues ha generado una gran nivel de debate sobre su alcance e implicaciones dentro de las diversas industrias (Raufflet, Lozano, Duque y de la Torre, 2012; Padilla et al., 2017) proponiendo cambios en las prácticas 
empresariales de manera que estas cambien direccionamiento hacia la sociedad, respondiendo a las exigencias de las comunidades que piden empresas más consientes con el impacto de sus actividades en la sociedad y en el medio ambiente (Solarte y Duque, 2017).

En este sentido, se aporta que la RSE es una obligación que poseen las empresas con relación al impacto que estas llegan a generar en su entorno y como estas tienen un importante efecto sobre las dimensiones social, económica y ambiental (Ramírez et al., 2018). Del mismo modo, esta busca garantizar que el impacto positivo equitativo, sustentable y que cause el menor impacto posible a las llamadas partes interesadas. Ciertamente la RSE implica que estas sean conscientes sobre el papel que tienen dentro un todo más complejo donde las organizaciones, al ser un fenómeno humano se involucren con las comunidades para aporta a las construcciones de una nueva sociedad (Pérez, Espinoza y Peralta, 2016; Castro, et al., 2019).

De esta manera, se convierte en un apunte al cambio, al desarrollo y a la calidad de las organizaciones que a la larga permite crear una percepción más humana de los consumidores, lo cual permitirá que la organización como marca tenga un reconocimiento positivo y una mayor aceptación.

Asimismo, en el libro verde se hacen mención a dos importantes dimensiones de la RSE (Salazar, Hidalgo y Manríquez, 2017; Abreo, Pinzón y Chacón, 2018); los cuales se enfocan a lo interno, referido a prácticas que se llevan a cabo dentro de la organización (Ortiz y Bandeira, 2015) y la dimensión externa, referida al impacto que las acciones tomadas por la organización tienen en el entorno de esta (Chumaceiro, Hernández, Yori y Ziritt, 2013).

A partir de aquí, el Centro para la Acción de la Responsabilidad Social Empresarial en Guatemala [Centra RSE] (2006) citado en De Grazia (2006) propone la pirámide de la responsabilidad social empresarial como medio de apoyo a las organizaciones que busquen implementarla; priorizando áreas de acción referidas a esta responsabilidad social empresarial. En esta pirámide se establecen los niveles de los criterios para diseñar políticas funcionales direccionadas al cambio sostenible y responsable:

Figura 1. Pirámide de la responsabilidad social empresarial

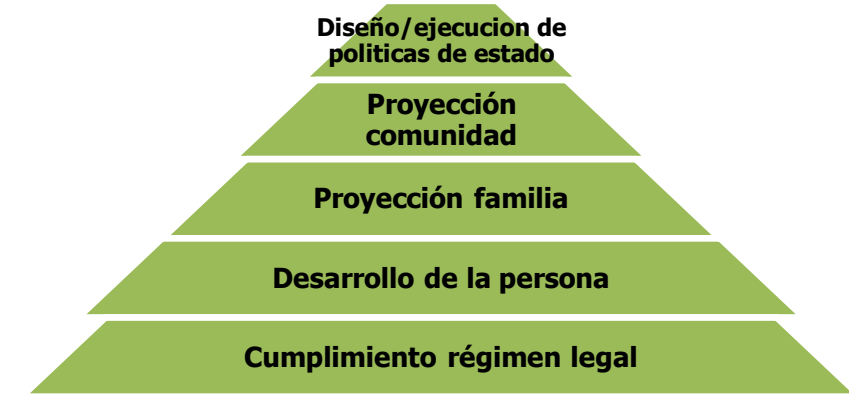

Fuente: Elaboración propia de autores basado en CENTRA RSE (2006). 
Autores como Barros Retamozo y González (2015) aportan que las políticas de estado permiten que las acciones vayan acorde a los planteamientos legales para responder a las proyecciones comunitarias. Así mismo generando un involucramiento con las familias, creando un espacio de confianza entre empresapersona para promover el desarrollo de las personas dentro y fuera de esta, siempre cumpliendo con los estándares legales requeridos, que fungen como la base de las acciones en todo momento. En este sentido, se reconoce que dentro de la responsabilidad social empresarial existen los llamados grupos de interés (Stakeholders); los cuales se direccionan a las partes interesadas o afectadas por las actividades (Freeman, 2010). Entre los grupos de interés se pueden mencionar a los trabajadores o las comunidades los cuales establecen sus intereses hacia la relación de las organizaciones son las temáticas políticas, sociales o ambientales. Dentro del presente artículo se realiza un énfasis a la relación entre la comunidad, las empresas y el medio ambiente al reconocer el impacto que se tiene sobre esta.

\section{Del cambio climático a la Ecoeficiencia}

Por muchos años se pensó que el cambio climático era un hecho ficticio sin soporte científico hasta que en los años 70 la comunidad científica comenzó a preocuparse por los constantes cambios de clima que sucedían para esa época, comenzando a hacer las primeras predicciones sobre el calentamiento global (Peterson, Connolley y Fleck, 2008; Deryugina, 2013). Con base a ello, ciertas décadas después los gobiernos del mundo apoyados en esta comunidad científica llevan a cabo en 1994 la Convención Marco de las Naciones Unidas sobre el Cambio Climático la cual se encaminó a estabilizar los niveles de contaminación generados a nivel mundial estableciendo un concepto de cambio climático que lo comprende desde la actividad humana que altera la composición de la atmosfera mundial (Oscullo y Haro, 2016).

A partir de estos primeros pasos se generan nuevos documentos como el Protocolo De Kioto de la Convención Marco de las Naciones Unidas sobre el Cambio Climático en 1998 hasta llegar al 2019, donde no solo existen rigurosas regulaciones sobre los temas ambientales, si no que se han generado modelos y propuestas que buscan mantener la rentabilidad y competitividad de la industria sin dejar a un lado los compromisos adquiridos en años anteriores.

Dentro de estos procesos se destaca la Ecoeficiencia (Deryugina, 2013), término que se atribuye a la relación existente entre el valor de un producto y/o servicio y los impactos medioambientales derivados de todo el proceso desde su comienzo hasta que este deja de ser utilizado (Durán, Calderón y Montero, 2018). Este proceso de Ecoeficiencia trae como resultado la vinculación de la conciencia ambiental dentro de las organizaciones, de manera que se logre tener el mayor margen de producción y ganancias, con el menor consumo de recursos y energía y así reducir el impacto sobre el medio ambiente (Carmelo, 2018). 
En tal sentido y basado en Rincón y Wellens (2011) se presentan los cinco principios de la Ecoeficiencia:

Figura 2. Principios de la eco-eficiencia

\section{Consumo de agua por unidad de valor agregado neto}

2. Requerimientos energéticos por unidad de valor agregado neto

3. Contribución al calentamiento global por unidad de valor agregado neto

4. Dependencia de sustancias que deterioran la capa de ozono por unidad de valor agregado neto

5. Residuos sólidos generados por unidad de valor agregado neto

Fuente: Rincón y Wellens (2011).

Se destaca que al implementar la Ecoeficiencia dentro de una organización implica plantear una iniciativa de valor pues al promover el desarrollo socioeconómico con un bajo coste de recursos (Vallejo y Cabrera, 2017), las mismas empresas se convierten en lideres dentro de su sector al ser estas las que generan una verdadera innovación (Sánchez, 2011). Aquí se plantea un interesante propuesto al afirmar que el daño ambiental proviene de los procesos para lograr los objetivos y no de los objetivos, por lo que son estos los que deben ser mejorados y trabajados (Cantú, 2015).

Se aporta que el empleo de la Ecoeficiencia permite a que las organizaciones sean vistas como parte de un todo en los sistemas naturales (González y Pérez, 2011), por lo que los procesos para su desarrollo y administración deberían poseer un enfoque sobre los componentes de la industria y la biosfera, la relación del flujo de la materia con el sistema industrial y considerar la tecnología como medio de transición para el logro de la no sustentabilidad (Carrillo, 2009).

Actualmente existen diversos enfoques al momento de medir la Ecoeficiencia, pero uno de los más representativos es el brindado por Desimone y Popoff (2000):

- Reducción del derroche de recursos apoyándose en la mejora continua.

- Disminución del volumen y toxicidad de los residuos.

- Reducción del consumo energético y emisiones contaminantes.

- Disminución de los riesgos de incumplimiento de leyes y se mejoran las relaciones de la comunidad y la empresa. 
En este sentido, se considera que un punto fundamental para lograr la Ecoeficiencia se relaciona con el empleo de las llamadas Mejores Tecnologías Disponible (MTD), término que se direcciona al uso de los equipamientos tecnológicos más avanzados y que su uso permite optimizar de la manera más adecuada los procesos de una organización. Ciertamente se ha reconocido el uso de las MTD para la reducción del impacto ambiental, ligado al rol ecológico que buscan tomar las nuevas tecnologías en la actualidad (Jiang et al., 2012)

\section{Método}

El presente estudio se desarrolló desde un enfoque cuantitativo, el cual permitió el análisis de resultados numéricos para su interpretación como procesos humanos. Del mismo modo, se direccionó desde la investigación descriptiva y exploratoria, para lograr identificar si las pequeñas y medianas empresas (Pymes) textiles, ubicadas en el departamento del Atlántico cumplen con las claves de la Ecoeficiencia. El diseño empleado fue de campo, no experimental y transversal pues la investigación requirió de realizar una medición única en el lugar de origen del fenómeno estudiado, pero sin llevar a cabo cualquier manipulación de las variables objeto de estudio dentro de la muestra seleccionada.

Este estudio se aplicó a los gerentes, administradores y jefes de producción de las PYMES textiles del departamento del Atlántico, Colombia. La muestra de la investigación es de tipo no probabilística (Otzen y Manterola, 2017), para la selección de esta, se consultaron las bases de datos de la Cámara de Comercio de Barranquilla, en donde se identificaron las pymes textiles ubicadas en el departamento del Atlántico, legalmente constituidas y, a partir de allí, se seleccionaron de manera aleatoria 53 empresas.

Las pymes elegidas fueron contactadas vía telefónica y, previo consentimiento informado, se les fue aplicado el instrumento, al cual dieron respuesta de manera efectiva. Para llevar a cabo el mencionado proceso de recolección de la data, el presente artículo se apoyó en el uso de un cuestionario cerrado con opciones dicotómicas (Si o No), el cual se configuró a partir de los principios básicos de la Ecoeficiencia, estructurando de esta manera seis preguntas clave que permiten evidencias el grado de compromiso y responsabilidad ambiental de las empresas seleccionadas como muestra en este estudio. La distribución de las preguntas del cuestionario responde de la siguiente manera a las claves de la Ecoeficiencia:

Tabla 1. Distribución del instrumento

\begin{tabular}{l|c}
\hline \multicolumn{1}{|c}{ Indicador } & İtem \\
\hline Reducción del derroche de recursos apoyándose en la mejora continua. & Ítem 1 y 5 \\
\hline Disminución del volumen y toxicidad de los residuos. & Ítem 2 \\
\hline Reducción del consumo energético y emisiones contaminantes. & Ítem 3 y 4 \\
\hline $\begin{array}{l}\text { Disminución de los riesgos de incumplimiento de leyes y se mejoran las relaciones de la } \\
\text { comunidad y la empresa. }\end{array}$ & Ítem 6 \\
\hline
\end{tabular}

Fuente: Elaboración Propia de autores (2019) 
Se destaca que este cuestionario se basa en los cinco principios de la Ecoeficiencia que son enumerados por Rincón y Wellens (2011). Este instrumento fue validado por un grupo de tres expertos en el área de sostenibilidad empresarial y responsabilidad social corporativa. Del mismo modo, a este instrumento se le aplico un proceso para determinar su confiabilidad a través del alfa de Cronbach la cual en este caso fue de 0,67 por lo que se considera como un valor aceptable en estudios de índole social (Oviedo y Arias, 2005). Se destaca que los datos fueron procesados por medio del programa estadístico SPSS.

\section{Resultados}

En esta sección se estructuran los resultados del cuestionario aplicado a los representantes de las 53 empresas seleccionadas como muestra en esta investigación, los cuales se estructuran a partir de cada una de las seis preguntas claves que les fueron formuladas durante la etapa de recolección. Una de las claves de la Ecoeficiencia es el control de los despilfarros de los recursos mediante la mejora continua; de acuerdo con el estudio el $76 \%$ de las pymes textiles participantes efectúa estos procesos de control de despilfarro; sin embargo, el 24\% restante afirma no llevar a cabo tales procesos. (Gráfico 1).

\section{Gráfico 1. Control de despilfarros}

¿La empresa controla el despilfarro de los recursos mediante la mejora continua?

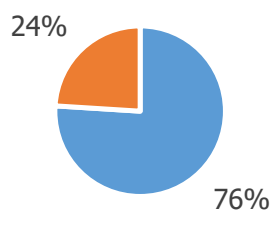

$=\mathrm{Si}=$ No

Cuando las empresas no tienen un control preciso sobre sus desperdicios no solo corren con el riesgo de que aumenten sus costos de producción, sino de que se disminuya de manera sustancial su rendimiento y la percepción de valor que poseen sus clientes en torno a los productos y servicios que ofrece, esto último puede explicarse de la siguiente manera: si los clientes perciben que la empresa no es consciente de reciclar, y reutilizar sus residuos de producción o de establecer técnicas para disminuir sus despilfarros, podrían preferir a empresas que presten los mismos servicios o productos pero cuya producción sea más amigable con la naturaleza, en aras de apoyar a compañías ambientalmente responsables y así, disminuir la huella ambiental industrial. Por fortuna, la mayoría de las compañías objeto de estudio, afirman tener un control preciso de sus despilfarros. 
Ahora bien, las empresas textiles utilizan diversos insumos químicos considerados como peligrosos en su proceso de producción, los cuales de no ser gestionados de la manera adecuada pueden constituirse como residuos altamente contaminantes para el medio ambiente; de allí la importancia de que las empresas textiles se esfuercen por disminuir el volumen y cambiar algunos insumos químicos que utilizan en la manufactura de sus productos por sustitutos con menor nivel de toxicidad. Se preguntó a las pymes textiles objeto de estudio si en la actualidad se esfuerzan por disminuir el volumen y el nivel de toxicidad de sus residuos, los resultados dejan en claro que la mayoría de estas entidades, representada por el $56 \%$ no llevan a cabo acciones para disminuir el volumen ni la peligrosidad de sus residuos y que, solo el $43 \%$ de estas empresas desarrolla este tipo de acciones. (Gráfico 2).

\section{Gráfico 2. Disminución volumen y toxicidad de residuos}

¿La empresa se esfuerza por dismuir el volumen y toxicidad de residuos?

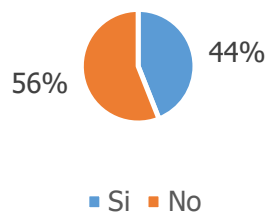

El hecho de que estas empresas no gestionen de la manera adecuada sus residuos, más aún si estos son peligrosos (sustancias químicas), se constituye como un riesgo evidente para el medio ambiente y para los stakeholders externos, principalmente la comunidad. A partir de esta falta de control, los residuos pueden alterar el equilibrio de los ecosistemas, contaminar las cuencas hídricas y afectar de manera profunda la salud de los animales y seres humanos. Otro de los cuestionamientos que se expusieron en el instrumento de investigación se basó en indagar si estas empresas controlaban su consumo de energía y emisiones contaminantes por medio de prácticas de producción limpia; el $72 \%$ de las pymes participantes dio una respuesta afirmativa; el $28 \%$ de las pymes restantes, expresó que no llevaban a cabo prácticas de producción limpias para el control de dichos aspectos (Gráfico 3).

\section{Gráfico 3. Prácticas de Producción limpia}

¿En la empresa se controla el consumo de energía y emisiones contaminantes por medio de practicas de producción limpia?

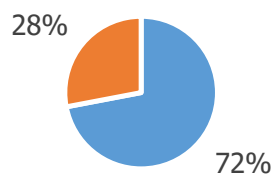

$$
-\mathrm{Si}=\text { No }
$$


Seguidamente se preguntó a las empresas si en la actualidad incorpora MTD para optimizar su producción; el $72 \%$ niega implementar este tipo de procesos; mientras que el $28 \%$ restante asegura si ejecutarlos. (Gráfico 4).

Gráfico 4. MTD para optimizar producción

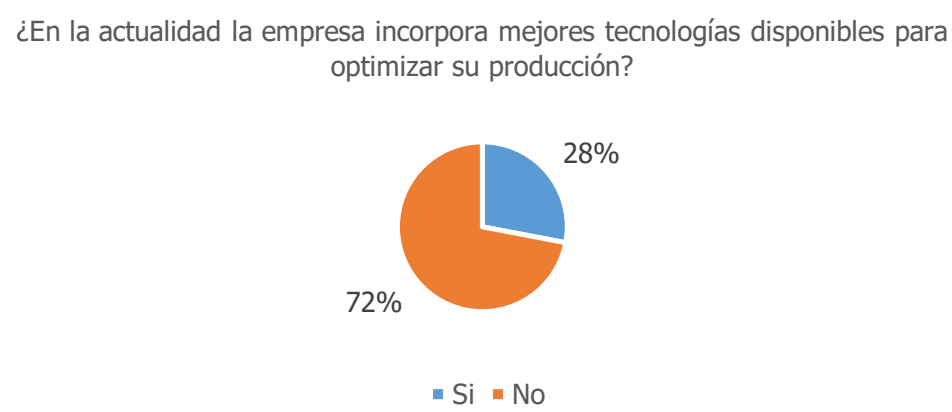

Las MTD, además, contribuyen a disminuir el impacto ambiental a través de la prevención de la contaminación y el diseño de productos ecológicos, lo que crea valor para las partes de interés y posiciona a la empresa como ambientalmente responsable.

Otro punto importante, que se estudió a partir del instrumento, se centra en el desarrollo de estrategias orientadas a reducir costos en el consumo de recursos (energéticos) y materias primas; muchas empresas no cuentan con el capital económico para incorporar prácticas de producción limpia o MTD, sin embargo, pueden diseñar e incorporar otro tipo de estrategias que les permita minimizar el consumo de recursos, más aun si se trata de aquellos categorizados como no renovables como la energía eléctrica convencional; según los resultados del estudio, el 56\% de las pymes participantes afirma que no desarrolla citadas estrategias; mientras que el $44 \%$ restante asegura si ejecuta este tipo de estrategias. (Gráfico 5)

\section{Gráfico 5. Estrategias reducción consumo}

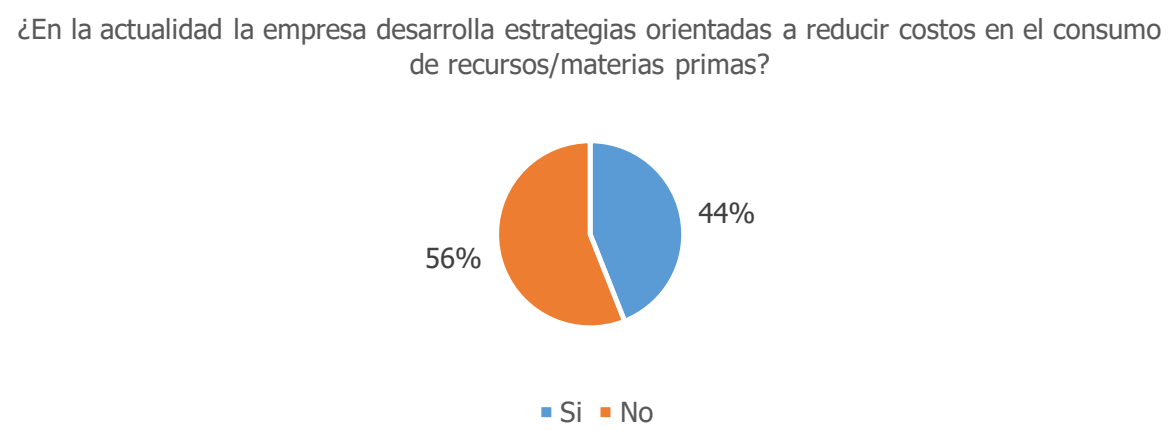

Finalmente, se preguntó a las empresas si consideraban que aumentando la seguridad ambiental podrían generar valor para sus partes de interés y aumentar su credibilidad en el mercado; el $84 \%$ estuvo 
de acuerdo con esta afirmación; mientras que el 16\% ignora que estas prácticas surtan beneficios para su operación. (Gráfico 6)

\section{Gráfico 6. Seguridad Ambiental para crear valor}

¿Considera que aumentando la seguridad ambiental podrá generar valor para sus partes de interes y aumental su credibilidad en el mercado?

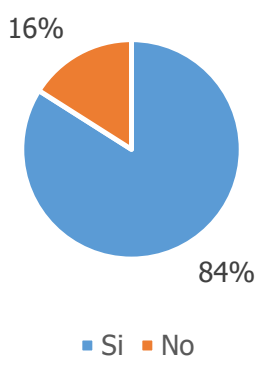

De esta manera, se observa que un gran porcentaje de las organizaciones que forman parte de la muestra reconocen el gran valor que genera la mejoría de la seguridad ambiental para las partes interesadas y así mejorar su credibilidad en el mercado.

\section{Discusiones}

A partir de los resultados del trabajo de campo, puede afirmarse que la mayoría de las pymes textiles ubicadas en el departamento del Atlántico no son empresas eco-eficientes; tal aseveración se sustenta en que, si bien la mayoría de las empresas asegura controlar sus despilfarros, estas no llevan a cabo procesos que les permitan disminuir el volumen y el grado de toxicidad de los insumos que utilizan en su producción, así pues, de poco sirve que una empresa regule las pérdidas de materiales, sino controla por ejemplo, el uso de sus materias primas o la cantidad de desechos peligrosos que dispone en el medio ambiente.

En consonancia con lo anterior, Rincón (2001) manifiesta que las empresas no pueden ser competitivas mientras no eliminen sus desperdicios y no trabajen con parámetros de productividad; pero, así mismo, esta competitividad depende de que las compañías minimicen el tiempo y la cantidad de recursos que utilizan en su producción; en este orden de ideas, las pymes textiles objeto de estudio, tienen grandes retos para ser competitivas a nivel nacional e internacional, puesto que, según el estudio, la mayoría de estas empresas no cuentan con estrategias orientadas a reducir el consumo de recursos (energéticos) y materias primas, lo cual conlleva a altos costos de producción y, por consiguiente, a aumentar la huella ambiental de su operación.

El consumo desmedido de energía, de acuerdo con Blanco y Arce (2012) produce grandes impactos ambientales, ya que, algunas centrales productoras de este recurso no renovable (Al menos en Colombia, por su plantas de generación convencional), utilizan procesos de combustión de los derivados de petróleo 
Remedios Pitre Redondo, Santander De la Ossa Guerra, Hugo Hernández Palma

para generarlo; así mismo, Duart (2007) manifiesta que el uso de la energía eléctrica en la industria contribuye al aumento en las emisiones de $\mathrm{CO} 2$, situación que tiene graves repercusiones sobre el calentamiento global y al aumento de las tasas de contaminación atmosférica. De igual modo, el consumo descontrolado de materias primas no solo genera una sobreexplotación de los recursos naturales, sino que incrementa la tasa de despilfarros y residuos que pueden contaminar el medio ambiente.

A partir de lo anterior, las empresas textiles deben reconocer la importancia de reducir el consumo de energía, según Campos et al. (2008) citado en Del Pilar et. al., (2013), "Las empresas industriales pueden lograr ahorros de energía de hasta un 40\%, algunos sin inversión de capital, mediante la aplicación de métodos de gestión energética" (p. 115); pero así mismo la importancia de reducir al máximo la utilización de materias primas no renovables y de incursionar en materiales sustitutos que cumplan con la misma función a un costo ambiental más bajo (Cañizares y Jurado, 2019).

Dentro del cuestionario se trabajó sobre la aplicación de las MTD para reducir el consumo de recursos, donde se encontró un bajo porcentaje representativo de su aplicación a nivel organizacional. De este modo, se aporta que las prácticas de producción limpias, más allá de evitar la emisión de residuos y de minimizar el uso indiscriminado de recursos energéticos, según Van y Herrera (2007) permiten la optimización y mejoramiento de procesos empresariales, promueven la innovación tecnológica (ecodiseño), la sostenibilidad e incluyen el concepto de ciclo vida como orientación de la gestión ambiental empresarial.

De la mano de las prácticas de producción limpia, surgen las MTD o Mejores Tecnologías Disponibles, las cuales, según Evrard et. al., (2016) permiten la reducción del uso de recursos finitos y, así mismo la efectividad en el uso de materias primas, para beneficiar a la empresa, el consumidor y la sociedad en general. Estos procesos de reducción y optimización, de acuerdo con Serna (2010) pueden levarse a cabo a partir de que las empresas determinen los sistemas y equipos que permitan la disminución de consumos, costos energéticos y costos ambientales; estos sistemas son reconocidos en el medio como las Mejores Tecnologías Disponibles (MTD) y su propósito es reducir al máximo los consumos, los despilfarros y los residuos de la producción.

Si bien estas MTD ofrecen grandes beneficios a las empresas, en el caso de las pymes textiles del departamento del Atlántico no han sido implementadas a cabalidad, de hecho el $72 \%$ de estas empresa expresa que en la actualidad no incorpora este tipo de tecnología a su operación; las razones no se especifican en el estudio, sin embargo, se estima que estas empresas no acuden a las MTD porque no están dispuestas a invertir recursos económicos o, así mismo, porque no ven su realmente utilidad, dejando de lado los grandes ahorros que estas MTD pueden generarles. Lo mencionado se enlaza con la pregunta direccionada a saber si se reducían los índices de consumos de materia prima, donde las respuestas fueron dividas. 
De allí la importancia de que las compañías objeto de estudio, hagan un uso consiente de sus materias primas, creen una cultura de reducción, reutilización y reciclaje, además, reemplacen los insumos nocivos para el medio ambiente, como son las tintas con base química, por elementos biodegradables, los cuales no solo les ayudarán a prevenir enfermedades de su fuerza laboral y la disminución de costes, sino que permitirán la salvaguarda del medio ambiente y la salud pública.

A su vez, se percibe que las organizaciones perciben el valor de mejorar la seguridad ambiental como herramienta para su relación con los grupos de interés y su reputación en el mercado. Cuando las empresas están enajenadas de los beneficios que pueden percibir al ser ambientalmente responsables, difícilmente se direccionaran a modificar sus prácticas, procesos de producción y tecnologías, ya que esto supone para ellas más gastos que utilidades; sin embargo, según Pérez y Camacho (2013) cuando las empresas logran ser reconocidas en el medio como una entidad con alto sentido de responsabilidad social y ambiental, esta puede mantener e incrementar su rentabilidad, puesto que se apalanca su imagen, posicionamiento y trascendencia de la marca en el mercado.

Es claro que, la mayoría de las empresas participantes en el estudio saben que, aumentando la seguridad ambiental, podrían generar valor para sus partes de interés $y$, de este modo, incrementar su credibilidad en el mercado; no obstante, son evidentes los grandes obstáculos que estas entidades tienen que sobrepasar para ser empresas ambientalmente responsables, dado que sus procesos y condiciones actuales están yendo en contravía de las crecientes exigencias en la legislación ambiental y los requerimientos de los consumidores "verdes" actuales. A partir de lo anterior, las pymes textiles deben implementar herramientas de gestión de Ecoeficiencia que les permitan diagnosticar las oportunidades de mejora ambiental, optimizar el consumo energético y de materias primas, establecer incluso medidas de ahorro de agua, tales como las auditorías ambientales, los sistemas de gestión medio ambiental ISO 14001 y programas de contabilidad ambiental.

\section{Conclusiones}

En primera instancia, se reconoce que la Responsabilidad Social Empresarial es un elemento de cambio dentro de las dinámicas empresariales de la actualidad; la cual brinda la oportunidad de direccionar a las organizaciones a convertirse entes más humanos, los cuales reconocen su importante papel dentro de la sociedad y de las comunidades como elemento promotor del crecimiento y del desarrollo local y regional.

De este modo, la vinculación a la Ecoeficiencia como proceso de cambio se presenta como una interesante propuesta para alcanzar altos estándares de calidad y de competitividad de las empresas vinculando estos objetivos con las necesidades del medioambiente, que, si bien en el pasado parecía ideas contrarias, hoy en día se comprueba que ambas van de la mano a través de una relación de sostenibilidad e innovación. De esta manera, al ser el sector textil el generador uno de los recursos con 
menor índice de reutilización en el mundo, se hace fundamental que esta vinculación con procesos de gestión sostenible y Ecoeficiencia sean claros y constantes, apuntando a una internalización de los procesos responsabilidad social, apoyándose en el estado, las familias, las personas y las leyes para su constante evolución y desarrollo.

Puede visualizarse en el mercado actual un compromiso creciente por parte de las empresas por contribuir a la sostenibilidad ambiental; estos avances además de tener un impacto positivo en la naturaleza, en la medida que se disminuye la contaminación, también tiene una incidencia en la mitigación de enfermedades que perjudican a los stakeholders externos y, cuyo foco son los residuos de la actividad industrial; sin embargo, al efectuar el estudio en las pymes textiles del departamento de Atlántico, Colombia, tal compromiso por la preservación y sostenibilidad ambiental es insuficiente, en primer lugar porque sus acciones son superfluas y estas direccionadas a reparar problemáticas coyunturales.

En segundo lugar, porque se centran más en desarrollar acciones para su propio beneficio que para beneficio ambiental, por ejemplo, estas empresas reducen la cantidad de despilfarros para ahorrar recursos y disminuir costos, pero, no reducen el volumen y toxicidad de los insumos que utilizan y que luego se convierten en residuos potencialmente peligrosos para el medio ambiente.

Los resultados obtenidos permiten observar que si bien las empresas reconocen la importancia de emplear mecanismos de seguridad ambiental en sus procesos y buscan disminuir el derroche de recursos, aún deben trabajar de mejor manera la reducción de materia prima, contaminación y recursos energéticos que se consumen dentro de estas. Al igual en el empleo de las mejores tecnologías disponibles como base de apoyo para la consecución de estas metas y puntos clave de la Ecoeficiencia.

\section{Referencias}

Abreo, A., Pinzón, B., \& Chacón, L. (2018). Programa responsabilidad social empresarial: empresa Global Service \& Business SAS. Aibi revista de investigación, administración e ingeniería, 6(2), 2-12. https://doi.org/10.15649/2346030X.479

Ballester, F., Iñíguez, C. Sáez, M., Pérez, S., Daponte, A. Ordóñez, J., Barceló, A. Taracido, M. Arribas, F. Bellido, J., Cambra, K., Cañada, A., y Guillén, J. (2003). Relación a corto plazo de la contaminación atmosférica y la mortalidad en 13 ciudades españolas. Medicina Clínica, 121(18), 684-689. https://doi.org/10.1016/S0025-7753(03)74063-9

Barros, M., Retamozo, M., y González, D. (2015). Responsabilidad social empresarial. Semilla DICE, (1), 81-87. http://www.unilibrebaq.edu.co/ojsinvestigacion/index.php/semilladice/article/view/448 
Blanco Orozco, N., y Arce-Díaz, E. (2012). El uso eficiente de la energía eléctrica en los ingenios azucareros como contribución al desarrollo sostenible de Nicaragua. Tecnología en Marcha, 26(3), 84-93. https://revistas.tec.ac.cr/index.php/tec_marcha/article/view/1520/1412

Cantú, P. (2015). Ecoeficiencia. Revista de divulgación científica y tecnológica de la Universidad Autónoma de Nuevo León, 18(71), 34. http://cienciauanl.uanl.mx/?p=3039

Cañizares, M., y Jurado, O. (2019). Las publicaciones sobre contabilidad de gestión con enfoque de ecoeficiencia: un desafío al futuro. Cofin Habana, 13(1). http://www.cofinhab.uh.cu/index.php/RCCF/article/view/326

Carmelo, J. (2018). Utilización de la web 2.0 como herramienta para la optimización de la ecoeficiencia de los alumnos del centro de Educación Técnico Productivo San Luis Gonzaga de la ciudad de Huánuco en el 2018 [Tesis de maestría, Universidad de Huánuco] Repositorio Institucional UDH. http://repositorio.udh.edu.pe/123456789/1765

Carrillo, G. (2009). Una revisión de los principios de la ecología industrial. Argumentos, 22(59). http://www.scielo.org.mx/scielo.php?script=sci_abstract\&pid=S0187$57952009000100009 \& \operatorname{lng}=e s \& n r m=i s o$

Castro, N., Garza, Z., González, L., y Zapata, Y. (2019). Dimensiones de la responsabilidad social empresarial: caso empresa procesadora de productos lácteos. Revista Internacional Administración \& Finanzas, 12(1), 69-78. https://www.theibfr.com/wpfb-file/riaf-v12n1-2019-5pdf/

Chumaceiro, A., Hernández, J., Yori, L., y Ziritt, G. (2013). Responsabilidad social empresarial y políticas $\begin{array}{lllll}\text { públicas. Revista de ciencias sociales, } & \text { XIX (2), 309-32 }\end{array}$ https://produccioncientificaluz.org/index.php/rcs/article/view/25624/26243

Colombia Productiva. (2012). Informe de Sostenibilidad sector sistema moda Colombia. https://www.colombiaproductiva.com/ptp-capacita/publicaciones/sectoriales/publicacionessistema-moda/informe-de-sostenibilidad-sistema-moda-2012

Cuartas, D., y Méndez, F. (2016). Cambio climático y salud: retos para Colombia. Revista de la Universidad Industrial de Santander. Salud, 48(4), 428-435. http://dx.doi.org/10.18273/revsal.v48n4-2016001

Custodio, M., \& De Oliveira, M. (2016). Eco-efficiency applied to the acquisition of everyday utensils in the brazilian federal administration. Revista de Direito Brasileira, 13, 19-39. 
Remedios Pitre Redondo, Santander De la Ossa Guerra, Hugo Hernández Palma

https://go.gale.com/ps/anonymous?id=GALE\%7CA597060901\&sid=googleScholar\&v=2.1\&it=r\&li nkaccess $=$ abs\&issn $=2237583 \times \& p=A O N E \& s w=w$

De Grazia, C. (2006, Abril). La pirámide de la Responsabilidad Social Empresarial. [Entrada de Blog]. https://carmelodegrazia.wordpress.com/2016/04/08/la-piramide-de-la-responsabilidad-socialempresarial/

Del Pilar-Castrillón, R., Ciro-Quispe, E., y González, A. (2013). Mejoramiento de la eficiencia energética en la industria del cemento por proceso húmedo a través de la implementación del sistema de gestión integral de la energía. Dyna, 801) 115-123. https://revistas.unal.edu.co/index.php/dyna/article/download/30656/43732

Deryugina, T. (2013). How do people update? The effects of local weather fluctuations on beliefs about global warming. Climatic change, 118(2), 397-416. https://doi.org/10.1007/s10584-012-0615-1

DeSimone, L. D., \& Popoff, F. (2000). Eco-efficiency: the business link to sustainable development. https://books.google.com.co/books?hl=es\&lr=\&id=SWF8wxxZGDYC\&oi=fnd\&pg=PR7\&dq=related :gs6SF_3IVbe5pM:scholar.google.com/\&ots=-Tx_FmKCeC\&sig=dPNaFiXs-

EGTZyLXKsuKzwJ5464\&redir_esc=y\#v=onepage\&q\&f=false

Duart-Belloque, V. (2007). Eficiencia y ahorro energético: contribución del sector de las tecnologías de la información. Universia Business Review, 116-127. https://journals.ucjc.edu/ubr/article/view/591

Durán, M., Calderón, E., y Montero, P. (2018). Ecoeficiencia y sus efectos sobre el desempeño económico de las empresas del Dow Jones Sustainability World Index 2016. Revista Prisma Social, (22), 271295. https://revistaprismasocial.es/article/view/2521

Duran, S., Fuenmayor, A., Sergio, C., y Hernández, R. (2016). Emprendimiento como proceso de responsabilidad social en instituciones de educación superior en Colombia y Venezuela. Desarrollo gerencial, $8(2)$. https://doi.org/10.17081/dege.8.2.2560

Eryuruk, S. (2012). Greening of the Textile and Clothing Industry. Fibres \& Textiles in Eastern Europe, 20(95), 22-27. http://fibtex.lodz.pl/article803.html

Evrard, D., Laforest, V., Villot, J., Gaucher, R. (2016). Best Available Techniques as a Sustainability Tool in Manufacturing: Case Study in the Dairy Sector. Procedia CIRP, 48, 520-525. https://doi.org/10.1016/j.procir.2016.03.098

Freeman, R. (2010). Strategic management: A stakeholder approach. Cambridge university press. 
Fundación Fórum Ambiental (2014). Ecoinnovación. http://www.forumambiental.org/es/category/sectorinnovacion/

González, M., y Pérez, M. 2011). La ecoeficiencia empresarial: su contribución al desarrollo local sostenible en los marcos de la globalización neoliberal. DELOS: Desarrollo Local Sostenible, 4(10), https://ideas.repec.org/a/erv/deloso/y2011i104.html

Intergovernmental Panel on Climate Change. (2007). Climate change 2007: The physical science basis. Agenda, 6(07), 333. https://www.ipcc.ch/report/ar4/wg1/

Jiang, C., Ren, Z., Tian, Y., \& Wang, K. (2012). Application of best available technologies on medical wastes disposal/treatment in China (with case study). Procedia Environmental Sciences, 16, 257265. https://doi.org/10.1016/j.proenv.2012.10.036

Maldonado, J. (2009). Ciudades y Contaminación Ambiental. Revista de Ingeniería, (30), 66-71. http://dx.doi.org/10.16924\%2Friua.v0i30.229

Mansour, S., Wittlinger, R., Alamdar, A., \& Burger, J. (2006). Ecoefficiency Analysis of Textile Coating Materials. Journal of the Iranian Chemical Society 3(4). 352-359. https://doi.org/10.1007/BF03245958

Ortiz, N., \& Bandeira, M. (2015). Responsabilidad social interna: entre la diferencia y el discurso en el escenario organizacional. Cuadernos EBAPE.BR, 13(2), 346-368. https://doi.org/10.1590/1679395115904

Oscullo, J., y Haro, L. (2016). Factor Anual de Emisión de CO2 Producido por el Parque Generador del Sistema Nacional Interconectado del Ecuador, mediante la Aplicación de la Metodología de la Convención Marco sobre el Cambio Climático UNFCCC, para el periodo 2009-2014. Revista Politécnica, $3 \pi(1)$, 61-61. https://revistapolitecnica.epn.edu.ec/ojs2/index.php/revista_politecnica2/article/view/664

Otzen, T., y Manterola, C. (2017). Técnicas de Muestreo sobre una Población a Estudio. International Journal of Morphology, 35(1), 227-232. http://dx.doi.org/10.4067/S0717-95022017000100037

Oviedo, H., y Arias, A. C. (2005). Aproximación al uso del coeficiente alfa de Cronbach. Revista colombiana de psiquiatría, 34(4), 572-580. http://psiquiatria.org.co/web/publicaciones/revistacolombiana-de-psiquiatria/volumen-34-n-4-ano-2005/metodologia/aproximacion-al-uso-delcoeficiente-alfa-de-cronbach/ 
Padilla, C., Arévalo, D., Bustamante, M., \& Vidal, C. (2017). Responsabilidad Social Empresarial y desempeño financiero en la industria del plástico en ecuador. Información tecnológica, 28(4), 93102. http://dx.doi.org/10.4067/S0718-07642017000400012

Parra, M., Duran, S., Marceles, V., Yarzagaray, J., Valenzuela, I., Payares, K., Alvarino, C., Navarro, N., Charris, P., y Ramírez, J. (2018). Factores del clima organizacional existentes en el área administrativa en empresas de Barranquilla. Revista ESPACIOS, 39(50). http://www.revistaespacios.com/a18v39n50/18395021.html

Pérez, G., y Camacho, M. (2013). Restaurantes Toks: estrategias de responsabilidad social. INNOVAR. Revista de Ciencias Administrativas y Sociales, 23, 141-155. https://revistas.unal.edu.co/index.php/innovar/article/download/40541/42373

Pérez, M., Espinoza, C., y Peralta, B. (2016). La responsabilidad social empresarial y su enfoque ambiental: una visión sostenible a futuro. Revista Universidad y Sociedad, 8(3), 169-178. https://rus.ucf.edu.cu/index.php/rus/article/view/430

Peterson, T., Connolley, W., \& Fleck, J. (2008). The Myth of the 1970s Global Cooling Scientific Consensus. Bull. Amer. Meteor. Soc, 89, 1325-1337. https://doi.org/10.1175/2008BAMS2370.1

Ramírez, F., Montoya, J., Rivera, D., Restrepo, C., y Osorio, L. (2018). De la responsabilidad social empresarial a la responsabilidad ambiental universitaria. Revista Lebret, (10), 133-155. https://doi.org/10.15332/rl.v0i10.2201

Raufflet, E., Lozano Aguilar, J., Duque, E., y \& De la Torre, C. (2012). Responsabilidad social empresarial. Pearson.

Rincón, E., y Wellens, A. (2011). Cálculo de indicadores de ecoeficiencia para dos empresas ladrilleras mexicanas. Revista internacional de contaminación ambiental, 27(4), 333-345. http://10.0.0.98/xmlui/handle/1/1623

Rincón-Parra, H. (2001). Calidad, Productividad y Costos: Análisis de relaciones entre estos tres $\begin{array}{lllll}\text { conceptos. } & \text { Actualidad }\end{array}$ https://doi.org/10.24054/01204211.v2.n2.2006.1923

Salazar, A., Hidalgo, J., y Manríquez, M. (2017). La responsabilidad social empresarial desde la percepción del capital humano. Estudio de un caso. Revista de Contabilidad-Spanish Accounting Review, 20(1), 36-46. https://doi.org/10.1016/j.rcsar.2016.01.001 
Sánchez, Y. (2011). Ecoeficiencia en la universidad hacia un desarrollo sostenible. Gestión en el Tercer Milenio, 14(27), 47-53. https://revistasinvestigacion.unmsm.edu.pe/index.php/administrativas/article/view/8855

Serna, C. (2010). Gestión energética empresarial una metodología para la reducción de consumo de energía. Producción + Limpia, 5(2). 107-126. http://doi.org/10.22507/pml

Solarte, M., \& Duque, J. (2017). Gestión humana y responsabilidad social empresarial: un enfoque estratégico para la vinculación de prácticas responsables a las organizaciones. Libre empresa, 9(1), 13-37. https://revistas.unilibre.edu.co/index.php/libreempresa/article/view/2965

Suárez, S. y Molina, E. (2014). El desarrollo industrial y su impacto en el medio ambiente. Revista Cubana de Higiene y Epidemiología, 52(3), 357-363. https://www.medigraphic.com/cgibin/new/resumen.cgi?IDARTICULO=59549

Superintendencia de Sociedades. (2013). Informe Desempeño del sector del sector textil-confección 20082012. https://www.supersociedades.gov.co/Documents/Informe-Sector-Textil-Oct152013.pdf

Torres, T., Barreto, I., y Rincón, J. (2015). Creencias y normas subjetivas como predictores de intención de comportamiento proambiental. Suma Psicológica, 12(2). 86-92. http://dx.doi.org/10.1016/j.sumpsi.2015.09.0030

Vallejo, G., y Cabrera, J. (2017). Determinación de la ecoeficiencia en el reciclaje de residuos sólidos en las empresas hoteleras de la ciudad de La Paz, BCS, a través de las variables económica y ambiental. UPIICSA. Investigación Interdisciplinaria, 3(2), 19-36. http://www.ruii.ipn.mx/index.php/RUII/article/view/49

Van-Hoof, B., y Herrera, C. (2007). La evolución y el futuro de la producción más limpia en Colombia. Revista de ingeniería universidad de los Andes, 26(1). 101-120. http://dx.doi.org/10.16924\%2Friua.v0i26.301

Vargas, F. (2005). La contaminación ambiental como factor determinante de la salud. Revista Española de Salud Pública, 79(2), 117-127. http://scielo.isciii.es/scielo.php?script=sci_arttext\&pid=S113557272005000200001 